\title{
An efficient iteratively reweighted L1-minimization for image reconstruction from compressed sensing
}

\author{
Zhengguang Xie, Hongjun Li, and Yunhua Li \\ School of Electronics and Information, Nantong University
}

\begin{abstract}
We proposed a simple and efficient iteratively reweighted algorithm to improve the recovery performance for image reconstruction from compressive sensing (CS). The numerical experiential results demonstrate that the new proposed method outperforms in image quality and computation complexity, compared with standard $l_{1}$-minimization and other iteratively reweighted $l_{1}$-algorithms when applying for image reconstruction from CS.

Keywords: Image reconstruction, Compressive sensing, $l_{1}$-Minimization, Reweighted algorithm.
\end{abstract}

\section{Introduction}

Compressive sensing theory presents [1-2] that a sparse signal can be reconstructed from a small number of random linear measurements using $l_{1}$ optimization (instead of $l_{0}$ optimization) algorithm, under some condition (such as mutual coherence (MC)[34], restricted isometry property/Condition (RIP or RIC) [5], or null space property (NSP) $[3,6]$ ). Recent studies indicate that the iteratively reweighted $l_{1}$-minimization does have an advantage over standard $l_{1}$-minimization in many situations [7-10] to find the sparest solution of an underdetermined linear system, which can be formulated as weighted $l_{1}$-problems $\left(W P_{1}\right)$ as follows.

$$
\begin{aligned}
& \qquad\left(W P_{1}\right) \quad \mathbf{x}^{(i)}=\min _{\mathbf{x} \in R^{N \times 1}}\left\|\mathbf{W}^{(i)} \mathbf{x}\right\|_{l_{1}} \text { subject to } \mathbf{A x}=\mathbf{y} \text { (1) } \\
& \text { here } \mathbf{w}^{(i)}=\operatorname{diag}\left(w^{(i)}\right) \text { and } w^{(i)}=\left(w_{1}^{(i)}, w_{2}^{(i)}, \cdots, w_{N}^{(i)}\right)^{T} \in \mathrm{R}^{N+} \text { (means positive real } \\
& \text { number) are the vector of weights determined by the previous iterate } \\
& \mathbf{x}^{(i-1)}=\left(x_{1}^{(i-1)}, \cdots, x_{j}^{(i-1)}, \cdots, x_{N}^{(i-1)}\right)^{T} \in \mathrm{R}^{N} .
\end{aligned}
$$

Mathematically speaking, the weight is used to drive $\mathbf{x}^{(i)}$ to it's the sparsest solution (the solution of $l_{0}$-minimization) via penalizing the components of $\mathbf{x}^{(i)}$ using minimizing the weighted $l_{1}$-norm. In other words, the target of $\left(W P_{1}\right)$ is to select a solution which is approximate to the solution of $l_{0}$-minimization from its all possible solutions. To this end, we need to specify a merit function for sparsity. Using such a

Zhengguang Xie, xie zg@126.com; Hongjun Li, corresponding author, xiezg@ntu.edu.cn; Yunhua Li, xiezg@ntu.edu.cn. School of Electronics and Information, Nantong University, China. 
function may drive the variable $\mathrm{x}$ to become sparse provided that a sparse solution exists. Clearly, there exist a vast number of merit functions for sparsity [11].

Recently, CS-based image/video sampling and compression has been studied in [1718]. These methods aim to reduce the number of CS measurements and thus improve the coding efficiency. In this paper work, we proposed a new and fast iteratively reweighted $l_{1}$-minimization algorithm for finding the sparest solution of an underdetermined linear system and extended our work to two dimensional signal (image) and measure the reconstruction quality and computation complexity, in comparison to classical $l_{1}$-minimization and other iteratively reweighted $l_{1}$ minimization algorithms.

\section{UNIFIEDSTRUCTURE OF REWEIGHTED ${ }^{l_{1}}$-MINIMIZATION}

Using an iterative algorithm to construct the weights $\mathbf{w}^{(i)}$ tends to allow for successively better estimation of the nonzero coefficient locations. The central idea of $\left(W P_{1}\right)$ is to define a weight $\mathbf{w}^{(i)}$ based on the previous iterate $\mathbf{x}^{(i-1)}$, solve $\left(W P_{1}\right)$ with the weight, and then use its solution to define a new weight $\mathbf{w}^{(i+1)}$. The structure of iteratively reweighted $l_{1}$-minimization is as follows.

1) Set the iteration count $i$ to zero and $w_{j}^{(0)}=1, j=1,2, \cdots, N$

2) Solve $\left(W P_{1}\right)$ (formula (1))

3) Terminate on convergence or when $i$ attains a specified maximum number of iterations $i_{\max }$. Otherwise, increment $i$.

4) Update the weights with the equation $\mathbf{W}^{(i)}=f\left(\mathbf{x}^{(i-1)}\right)$ and the then go to step 2.

The weight of a reweighted $l_{1}$-minimization is yielded by merit function as follows

$$
w_{j}^{(i+1)}=f\left(x_{j}^{(i)}\right)=\nabla g_{j}\left(\left|x_{j}^{(i)}\right|+\varepsilon^{(i)}\right)
$$

or by support set $\left(T_{S}\right)$

$$
w_{j}^{(i+1)}=\left\{\begin{array}{lc}
C_{1}, & \left|x_{j}^{(i)}\right| \in T_{S} \\
C_{0}, & \text { others }
\end{array}\right.
$$

here $g_{j}\left(\left|x_{j}^{(i)}\right|+\varepsilon^{(i)}\right)$ is a merit function, $\nabla$ is a gradient operator, $\varepsilon^{(i)}, C_{1}$ and $C_{0}$ are constant. This leads weighted $l_{1}$-minimization (1) to the approximation problem of $\left(\mathrm{P}_{0}\right)$.

For example, the function $\mathrm{G}_{\varepsilon}(\mathbf{x})=\sum \log \left(\left|x_{j}\right|+\varepsilon\right)$ was used by Gorodnitsky and

Rao [19] to design the FOCUSS algorithm, and E. J. Candès [7] to design reweighted $l_{1}$-minimization, for sparse signal reconstruction. Considering the limited space of this 
paper, we summarize the existing algorithms as follows, which are based on a merit function or support set for sparsity.

1)E. J. Candès [7](WL1FIX)

$$
g_{j}\left(\left|x_{j}\right|+\varepsilon\right)=\log \left(\left|x_{j}\right|+\varepsilon\right) ; \quad w_{j}^{(i+1)}=\frac{1}{\left|x_{j}^{(i)}\right|+\varepsilon^{(i)}}
$$

2) D. Wipf [8](WR2REG)

$$
g_{j}\left(\left|x_{j}\right|+\varepsilon\right)=\log \left(x_{j}^{2}+\varepsilon\right) ; \quad w_{j}^{(i+1)}=\frac{1}{\left(x_{j}^{(i)}\right)^{2}+\varepsilon^{(i)}}
$$

3) Y. Wang [11](ISD)

$w_{j}^{(i+1)}=\left\{\begin{array}{ll}1, & \left|x_{j}^{(i)}\right| \in T_{S} \\ 0, & \text { others }\end{array}, g_{j}\left(\left|x_{j}\right|+\varepsilon\right)\right.$ is a Support Set $\left(T_{S}\right)$

4) L. Qin [9]( RISD)

$w_{j}^{(i+1)}=\left\{\begin{array}{ll}\frac{1}{\left|x_{j}^{(i)}\right|}, & \left|x_{j}^{(i)}\right| \in T_{S} \\ \frac{1}{\varepsilon^{(i)}}, & \text { others }\end{array}, g_{j}\left(\left|x_{j}\right|+\varepsilon\right)\right.$ is also a Support Set $\left(T_{S}\right)$. Note that the support set of ISD and RISD is according to first jump rule $[9,11]$.

5) Y.-B. ZHAO [10]( WLP)

$$
g_{j}\left(\left|x_{j}\right|+\varepsilon\right)=\frac{1}{p} \sum_{j=1}^{N}\left(\left|x_{j}\right|+\varepsilon\right)^{p} ; \quad w_{j}^{(i+1)}=\frac{1}{\left.\left|x_{j}^{(i)}\right|+\varepsilon^{(i)}\right)^{1-p}}
$$

6) Y.-B. ZHAO $[10](\mathrm{NW} 1)$

$$
\begin{aligned}
\left.g_{j}\left|x_{j}\right|+\varepsilon\right)=\frac{1}{p} \sum_{j=1}^{N}\left[\left|x_{j}\right|+\varepsilon+\left(\left|x_{j}\right|+\varepsilon\right)^{p}\right] \\
w_{j}^{(i+1)}=\frac{p+\left(\left|x_{j}^{(i)}\right|+\varepsilon^{(i)}\right)^{1-p}}{\left(\left|x_{j}^{(i)}\right|+\varepsilon^{(i)}\right)^{1-p}\left[\left|x_{j}^{(i)}\right|+\varepsilon^{(i)}+\left(\left|x_{j}^{(i)}\right|+\varepsilon^{(i)}\right)^{p}\right]}
\end{aligned}
$$

7) Y.-B. ZHAO [10]( NW1)

$$
\begin{gathered}
g_{j}\left(\left|x_{j}\right|+\varepsilon\right)=\frac{1}{p} \sum_{j=1}^{N}\left[\left|x_{j}\right|+\varepsilon+\left(\left|x_{j}\right|+\varepsilon\right)^{q}\right]^{p} \\
w_{j}^{(i+1)}=\frac{q+(8)}{\left.\left(\left|x_{j}^{(i)}\right|+\varepsilon^{(i)}\right)^{1-q}\left[\left|x_{j}^{(i)}\right|+\varepsilon^{(i)}\right)^{1-q}+\left(\left|x_{j}^{(i)}\right|+\varepsilon^{(i)}\right)^{q}\right]^{1-p}}
\end{gathered}
$$

The existing iteratively reweighted $l_{1}$-minimization algorithms are based on a merit function or a support set, from which the weights are derived. Numerical experiments prove that the performance of all algorithms of this family is almost few different [10], which can be seen in numerical experiments section. To further improve the 
performance of this kind of algorithms, we propose a very simple and efficient algorithm.

\section{A SIMPLE METHOD PROPOSED}

Based on ISD [11], we proposed a simple algorithm which is outlined as follows. Input: A, $\mathbf{y}$, Initialize a set $\Lambda_{0}=\varnothing, i=0$. While $i<i_{\max }$ and the stopping criterion is not met, do

1) Update $\mathbf{x}^{(i)}$ according to

$$
\mathbf{x}^{(i)}=\min _{\mathbf{x}} \sum_{j \notin \Lambda_{i}}\left|x_{j}\right| \text { subject to } \mathbf{A x}=\mathbf{y}
$$

2) Terminate on convergence or when $i$ attains a specified maximum number of iterations $i_{\max }$. Otherwise, increment $i$.

3) Sort $\left\{\left|x_{j}^{(i-1)}\right|\right\}, j=1 \cdots N$ in descending order and assign subscripts of the largest $M / 2 \times i / i_{\max }$ ( $M$ is the number of measurements) to $\Lambda_{i}$, and the then go to step 1.

The interpretation of the above algorithm is as an iterative reweighted the algorithm with a " $0 / 1$ " weighting scheme. The nth largest signal coefficients (nth-large signals)are most likely to be identified as nonzero. For the purpose of allowing more sensitivity for identifying the remaining small nonzero signal coefficients (remaining small signals), the influence of nth-large signals should be omitted; while the influence of remaining small signals should be strengthen. Therefore, the weights of nth-large signals are set to ' 0 ' in the subsequent iteration, while the weights of remaining small signals are set to ' 1 '. The main reasons are that 1) among the nth-large signals, the probability to be nonzero entries is high, but isn't completely in proportion to their absolute value, 2) among the remaining small signals, the probability to be zero entries is high, but isn't completely is inversely proportional to their absolute value. It is often the case the nth largest signals coefficients include some zero entries and/or the remaining small signals have some nonzero entries. However, numerical results strongly suggest that the new method has a self-corrected capacity.

The advantage of our strategies are 1) no regularization parameter is needed, 2) the weights for all entries are ' 1 ' or ' 0 ', 3 ) its performance is better than the existing methods, in terms of both successful probability and complexity of sparse signal recovery.

\section{NUMERICAL EXPERIMENTS}

There are lots of merit functions or support sets for sparsity, based on which various reweighted $l_{1}$-methods can be constructed. This section is to compare these algorithms through numerical experiments. For limited space, we only compare their 
performances of the five algorithms (WL1FIX, WL2REG, ISD, RISD, Proposed), since the performance of WLP, NW1 and NW2 are almost the same as that of IRL1[10].

\subsection{Experimental setting and test platforms}

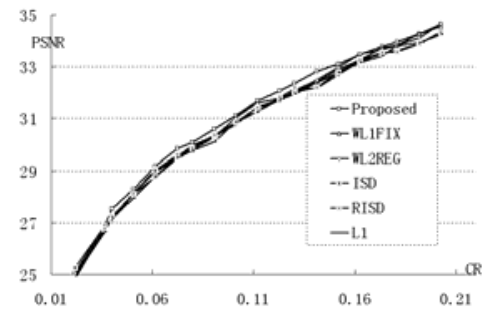

Fig.1 Comparison of the PSNR under the same Compression Ratio for "512 5512 Lena" Image for six algorithms

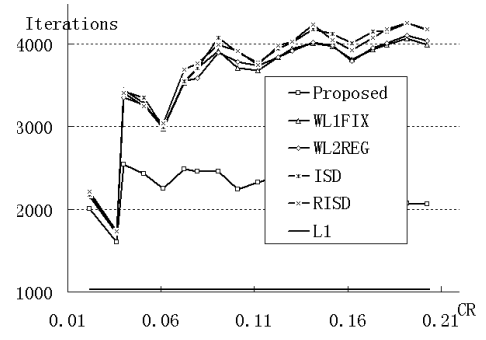

Fig.2: Comparison of the numbers of Iterations for " $512 \times 512$ Lena" Image for six algorithms

To compare these methods, we use " $512 \times 512$ Lena" as a test image. For every block, the number of measurements is decided by the following formula.

$$
M=\left\{\begin{array}{l}
0.7 \times 3 \times K, \delta \leq 10 \\
1.4 \times 3 \times K, \text { others }
\end{array}\right.
$$

where $\delta$ is the variance of the encoding block, and $K$ is the value of sparsity . For every algorithm, the sparsity $K$ is set from 2 to 20 , which are 19 tests in all. Every test is according the following step:

1) Divide the image into 16x16 blocks and then run two dimensions Discreet Cosine Transform (DCT).

2) Each block DCT coefficients convert to 1-D signal and then remove all but the largest $K$ entries from them (signal $\mathbf{x}$ ).

3) Encode the $K$-sparsity 256-entries signal by using Measurement matrix $\mathbf{A} \in R^{M \times 256}$ which is random Gaussian matrix generated by MATLAB. The measurements $\mathbf{y}$ is equal to $\mathbf{A x}$.

4) Signal reconstruct using one of the above five algorithms.

For all tested instances of $\mathbf{A x}=\mathbf{y}$, the selected iteratively reweighted algorithm was executed, at most 4 iterations, with the same parameters $\varepsilon^{(i)}$ (to be set as [7], only for WL2REG), and the initial point $\mathbf{x}^{(0)} \in R^{256 \times 1}$ (the initial value of $\mathbf{x}$ which is set to the solution of the $l_{1}$-minimization). Given a $K$-sparse solution $\mathbf{x}$ of $\mathbf{A x}=\mathbf{y}$, the algorithm claims to be successful in finding the $K$-sparse solution $\mathbf{x}$ if the solution 
$\mathbf{x}^{(i)}$ satisfies $\left\|\mathbf{x}^{(i)}-\mathbf{x}\right\|_{l_{\infty}} \leq 10^{-3}$. To solve these problems, we use CVX, a package for specifying and solving convex programs [20].

5) Each 1-D reconstructed signal convert to 2-D block DCT coefficients.

6) Invert 2-D DCT transform and piece the blocks together a reconstructed image.

\subsection{Experimental results}

To compare the performance, the compression ratio (CR) is defined as the ratio of measurement numbers and the raw data size.

$$
C R=\frac{M_{s}}{256 \times 256}
$$

here $M_{s}$ is the number of measurements for all $16 \times 16$ blocks according to (12). The compression quality is the peak signal-to-noise ratio (PSNR) is as follows.

$$
P S N R=10 \log _{10}\left\{\frac{512 \times 512 \times 255^{2}}{\sum_{w=1}^{512} \sum_{h=1}^{512}\left(x_{w, h}^{r e c}-x_{w, h}^{\text {org }}\right)^{2}}\right\}
$$

where the $x_{w, h}^{r e c}$ is the value of the reconstructed pixel of the location (value of the reconstructed pixel of the location $(w, h) ; x_{w, h}^{\text {org }}$ the value of the original pixel of the location $(w, h)$. Clearly, the main computational cost is solving weighted $l_{1}$ minimization problems, so we use the number of iteration to approximately estimate the computation complexity of all the algorithms.

Figure 1 shows that the PSNR of the image reconstructed by using the proposed algorithm is higher than any other existing iteratively reweighted L1-minimization algorithms, closely followed by the WL1FIX, WL2REG, ISD, RISD and L1minimization, whose average PSNR are $31.09 \mathrm{~dB}, 30.90 \mathrm{~dB}, 30.89 \mathrm{~dB}, 30.82 \mathrm{~dB}$, $30.79 \mathrm{~dB}, 30.75 \mathrm{~dB}$, respectively.

Figure 2 shows that the computation complexity of the image reconstructed by using the proposed algorithm is lower than any other existing iteratively reweighted L1minimization algorithms, closely followed by the WL1FIX, WL2REG, ISD, and RISD, whose average PSNR are 2242.63, 3560.74, 3566.73, 3667.11, 3667.58, respectively.

Figure 3and 4 show the CS reconstruction image. There exists block effect in image of figure 3. Image of figure 4 is comparable with the original image (figure 5). 


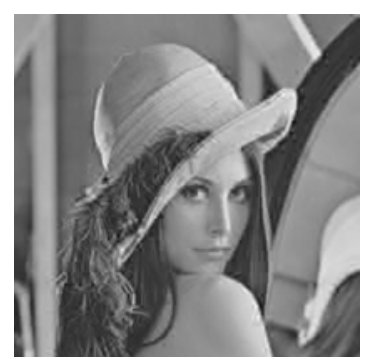

Fig.3. vision quality of CS reconstruction image when $\mathrm{K}=5, \quad \mathrm{M}=17$, $(\mathrm{PSNR}=28.93$, $\mathrm{CR}=0.061)$

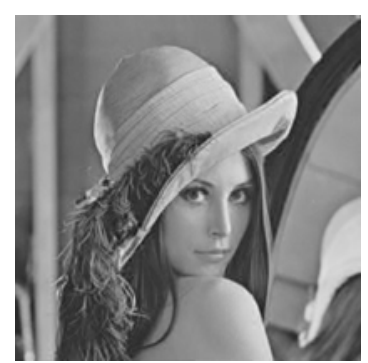

Fig.4. vision quality of CS reconstruction image when $\mathrm{K}=10, \quad \mathrm{M}=35$, $(\mathrm{PSNR}=32.20, \mathrm{CR}=0.13)$

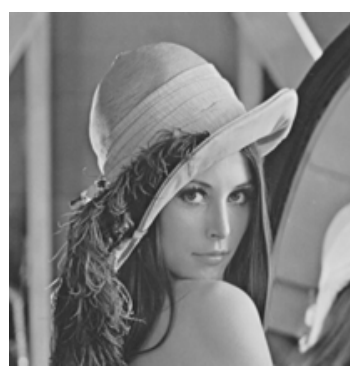

Fig. 5. original image

\section{CONCLUSION}

In summary, we compared both CS reconstruction image quality and computation complexity between the proposed algorithm and the existing reweighted L1minimization algorithms. The numerical experimental results demonstrate that the proposed algorithm outperforms the others.

\section{Acknowledgements}

This work was supported in part by the National Natural Science Foundation of China (61171077), the basic research programs of Chinese Department of Transportation (2011-319-813-510), the science and technology supporting plan (social development) of Jiangsu Province (BE2010686), joint tackle hard-nut problems in science and technology on traffic and transportation industry of state ministry of communications (2010-353-332-110), and innovation training program foundation of Nantong University (2010).

\section{References}

[1] D. L. Donoho, "Compressed sensing," Information Theory, IEEE Transactions on, vol. 52, pp. 1289-1306, 2006.

[2] E. J. Candes, et al., "Robust uncertainty principles: exact signal reconstruction from highly incomplete frequency information," Information Theory, IEEE Transactions on, vol. 52, pp. 489-509, 2006.

[3] D. Donoho and M. Elad, "Optimality sparse representation in general (nonorthogonal) dictionaries via $\ell 1$ minimization," Proc. Natl. Acad. Sci., vol. 100, pp. 2197-2202, 2003. 
[4] D. Donoho and X. Huo, "Uncertainty principles and ideal atomic decomposition," IEEE Trans. Inform. Theory, vol. 47, pp. 2845-2862, 1999.

[5] E. Candes and T. Tao, "Decoding by linear programming," IEEE Trans. Inform. Theory, vol. 51, pp. 4203-4215, 2005.

[6] Y. Zhang, "Theory of compressive sensing via $\ell 1$-mimimization: A Non-RIP analysis and extensions," Technical Report, Rice Univ., 2008.

[7] E. J. Candès, et al., "Enhancing sparsity by reweighted L1 minimization," Journal of Fourier Analysis and Applications vol. 14, pp. 877-905, 2008.

[8] D. Wipf and S. Nagarajan, "Iterative reweighted L1 and L2 methods for finding sparse solutions," Selected Topics in Signal Processing, IEEE Journal of, vol. 4, pp. 317-329, 2010.

[9] L. Qin, et al., "A new reweighted algorithm with support detection for compressed sensing," Signal Processing Letters, IEEE, vol. 19, pp. 419-422, 2012.

[10] Y.-B. ZHAO and D. LI, "Reweighted $\ell 1$-minimization for sparse solution to underdetermined linear systems," SIAM Journal on Optimization, vol. 22, pp. 1065-1088, 2012.

[11] Y. Wang and W. Yin, "Sparse signal reconstruction via iterative support detection," SIAM Journal on Imaging Sciences, vol. 3, pp. 462-491, 2010.

[12] R. Chartrand and Y. Wotao, "Iteratively reweighted algorithms for compressive sensing," in Acoustics, Speech and Signal Processing, 2008. ICASSP 2008. IEEE International Conference on, 2008, pp. 3869-3872.

[13] R. E. Carrillo and K. E. Barner, "Iteratively re-weighted least squares for sparse signal reconstruction from noisy measurements," in Information Sciences and Systems, 2009. CISS 2009. 43rd Annual Conference on, 2009, pp. 448-453.

[14] I. Daubechies, et al., "Iteratively reweighted least squares minimization for sparse recovery " Comm. Pure Appl. Math., vol. 63, pp. 1-38, 2010.

[15] A. K. Krishnaswamy, et al., "A simpler approach to weighted L1 minimization," in Acoustics, Speech and Signal Processing (ICASSP), 2012 IEEE International Conference on, 2012, pp. 3621-3624.

[16] P. Holland and R. Welsch, "Robust regression using iteratively reweighted least-squares," Commun. Stat. Theor. Methods, vol. A6, pp. 813-827, 1977.

[17] Y. Tsaig and D. L. Donoho, "Extensions of compressed sensing," Signal Processing Letters, IEEE, vol. 15, pp. 533-548, 2006.

[18] C. W. Deng, et al., "robust image compression based upon compressive sensing," presented at the IEEE Int. Conf. Multimedia and Expo., 2010.

[19] I. Gorodnitsky and B. Rao, "Sparse signal reconstruction from limited data using FOCUSS: A reweighted minimum norm algorithm," IEEE Trans. Signal Process, vol. 45, pp. 600-616, 1997.

[20] M. Grant and S. Boyd, "CVX: Matlab software for disciplined convex programming," 2011. 